\section{Suicidal intent as a risk factor for mortality in high-level falls: a comparative study of suicidal and accidental falls}

\author{
Byung Hee Kang, Kyoungwon Jung, Yo Huh \\ Division of Trauma Surgery, Department of Surgery, Ajou University School of Medicine, Suwon, Korea
}

Objective Suicide is a major issue in South Korea, and falling is a common method of suicide. Further, accidental falls are a common cause of death. However, whether suicidal falls differ from accidental falls is inconclusive. This study aimed to compare suicidal and accidental falls to identify risk factors for mortality.

Methods From March 2010 to December 2016, patients admitted to our hospital because of falls were reviewed retrospectively. Characteristics and outcomes were compared between suicide and accident groups. Injury distribution was compared using the Injury Severity Score and Abbreviated Injury Scales. Multivariate analysis was performed to identify risk factors, including suicide intent, for mortality.

Results Of 242 patients, 42 were included in the suicide group and 200 were included in the accident group. The suicide group showed higher fall heights and injuries of greater severity. The accident group was younger and included a higher number of men. The suicide group showed a higher mortality $(23.8 \%$ vs. $6.5 \%, P=0.001)$ and a higher proportion of injuries in the lower extremities or abdomen. In the multivariate analysis, Glasgow Coma Scale score (0.575 [0.433-0.764], $P<0.001)$, body mass index (1.638 [1.194-2.247], $P=0.002$ ), suicide intent (9.789 [1.026-93.404], $P=0.047)$ and Injury Severity Score $(1.091[1.000-1.190], P=0.049)$ were identified as risk factors for mortality.

Conclusion Suicidal falls were associated with poorer outcomes and a greater tendency to land feet first relative to accidental falls. Suicide intent was a risk factor for mortality.

Keywords Accidental falls; Suicide; Mortality; Risk factors

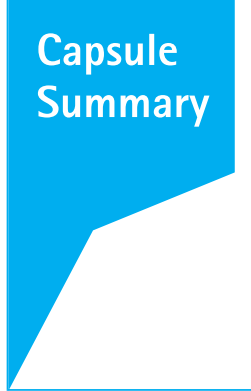

What is already known

Suicidal falls might be different from accidental falls in injury distribution, and it is still unknown whether clinical outcomes are different.

What is new in the current study

Suicidal falls have poor clinical outcome, and suicidal intent is a risk factor for mortality.
elSSN: 2383-4625

Received: 28 February 2020

Revised: 3 April 2020

Accepted: 6 April 2020

Correspondence to: Yo Huh Division of Trauma Surgery, Department of Surgery, Ajou University School of Medicine, 164 World cup-ro, Yeongtong-gu, Suwon 16499, Korea E-mail: ermdhuhyo@aumc.ac.kr ORCID

https://orcid.org/0000-0002-1220-1534

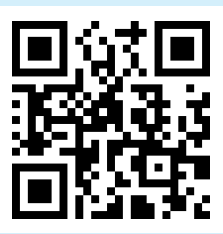

How to cite this article:

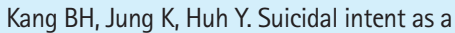
risk factor for mortality in high-level falls: a comparative study of suicidal and accidental falls. Clin Exp Emerg Med 2021;8(1):16-20. https://doi.org/10.15441/ceem.20.019

This is an Open Access article distributed under the terms of the Creative Commons Attribution Non-Commercial License (https:// creativecommons.org/licenses/by-nc/4.0/). 


\section{INTRODUCTION}

Suicide is the fifth most common cause of death and the most common cause of death in young adults in South Korea.' In 2016, the suicide attributable death rate was 25.6 per 100,000 population, which was in excess of the global rate of 10.6 per 100,000 population estimated by the World Health Organization that year. ${ }^{2}$ Despite efforts such as prevention campaigns and increased access to regional trauma centers, mortality due to suicide remains high. ${ }^{3-5}$ Falls are the second most commonly recorded method of suicide fatality, while accidental falls are the tenth most common cause of death in South Korea. ${ }^{6,7}$ Injury mechanisms are likely to be shared but distinguished by statistics for death rates.

Several previous studies have compared accidental and suicidal falls. ${ }^{8-11}$ In these reports, relative to accidental falls, suicidal falls were more likely to occur in younger female patients, and injury patterns appeared to differ between groups. However, whether suicidal intent is associated with poor outcomes remains unclear. Factors such as fall height, body mass index (BMI), age, and a combination of injuries, could affect outcomes, suggesting that they should be considered along with intent. ${ }^{12-14}$ Of these factors, fall height could require particular attention because falls from low heights are rarely fatal in adults..$^{15}$ This study compared suicidal and accidental falls and assessed fall associated risk factors, including suicide intent, for mortality.

Table 1. Demographic and clinical characteristics of patients in the suicidal and accidental fall groups

\begin{tabular}{lccr}
\hline & $\begin{array}{c}\text { Suicide } \\
(\mathrm{n}=42)\end{array}$ & $\begin{array}{c}\text { Accident } \\
(\mathrm{n}=200)\end{array}$ & P-value \\
\hline Age (yr) & $33(26-40)$ & $46(38-54)$ & $<0.001$ \\
Sex, male & $18(42.9)$ & $174(87.0)$ & $<0.001$ \\
Fall height (m) & $9.5(6.0-15.0)$ & $6.0(6.0-10.0)$ & $<0.001$ \\
Body mass index & $22.1(20.3-26.1)$ & $22.7(20.5-24.9)$ & 0.995 \\
Systolic blood pressure (mmHg) & $100(91-126)$ & $122(109-140)$ & $<0.001$ \\
Heart rate (beat/min) & $92(82-111)$ & $86(78-98)$ & 0.019 \\
Shock index & $0.91(0.69-1.18)$ & $0.70(0.59-0.87)$ & $<0.001$ \\
Lactate (mmol/L) & $4.29(2.42-5.10)$ & $2.78(1.87-3.90)$ & 0.004 \\
Glasgow Coma Scale & $15(8-15)$ & $15(14-15)$ & 0.015 \\
Injury Severity Score & $22(14-29)$ & $17(13-27)$ & 0.035 \\
AIS head & $3(2-3)$ & $3(2-3)$ & 0.333 \\
AIS face & $2(1-2)$ & $2(2-2)$ & 0.429 \\
AIS thorax & $3(3-3)$ & $3(3-3)$ & 0.748 \\
AIS abdomen & $2(2-3)$ & $2(2-3)$ & 0.273 \\
AIS extremities & $3(2-3)$ & $2(2-3)$ & 0.028 \\
AIS external & $1(1-1)$ & $1(1-1)$ & 0.900 \\
\hline
\end{tabular}

Values are presented as median (interquartile range) or number (\%). AIS, Abbreviated Injury Scale.

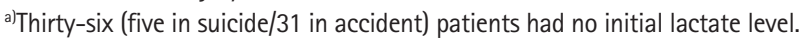

\section{METHODS}

This study was approved by the institutional review board at the institution with which the authors were affiliated (AJIRB-MEDMDB-19-331). Informed consent was waived by the board due to the observational nature of the study. Data regarding patients admitted to our hospital because of falls between March 2010 and December 2016 were reviewed. Patients were excluded if they were aged $<18$ years, had unknown suicide intent, or had died before admission. Because the minimum height of suicidal falls was $5 \mathrm{~m}$ in the database, patients with accidental fall heights of $<5 \mathrm{~m}$ were excluded, although major falls are usually from heights of $>6 \mathrm{~m}$.

Chi-squared test was performed to analyze categorical variables, and the Mann-Whitney U test was used to analyze continuous variables, following the use of the Kolmogorov-Smirnov test for normality assumption. Injury distribution was assessed based on the Abbreviated Injury Scale (AIS) and included injuries to the head, face, neck, thorax, abdomen, spine, and upper and lower extremities, and external injuries. In addition, patients were dichotomized into survivor and non-survivor groups. Multivariate analysis was performed using logistic regression via the forced entry method; variables that were statistically significant $(P<0.05)$ in the univariate analysis were selected as potential risk factors for mortality. Statistical analysis was performed using IBM SPSS Statistics ver. 23 (IBM Corp., Armonk, NY, USA).

\section{RESULTS}

\section{Suicidal vs. accidental falls}

Of 242 patients, 42 were included in the suicidal fall group and 200 were included in the accidental fall group (Table 1). Demo-

Table 2. Clinical outcomes of patients within the suicidal and accidental fall groups

\begin{tabular}{lccr}
\hline & Suicide $(\mathrm{n}=42)$ & Accident $(\mathrm{n}=200)$ & P-value \\
\hline $\begin{array}{l}\text { Mortality } \\
\text { Cause of death }\end{array}$ & $10(23.8)$ & $13(6.5)$ & 0.001 \\
$\quad$ Central nervous system & $2(20.0)$ & $8(61.5)$ & \\
$\quad$ Bleeding & $3(30.0)$ & $0(0.0)$ & \\
$\quad$ Multi-organ failure & $5(50.0)$ & $5(38.5)$ & \\
ICU LOS (day) & $3(0-8)$ & $2(0-7)$ & 0.239 \\
Hospital LOS (day) & $26(13-47)$ & $26(17-48)$ & 0.653 \\
24 hr pRBC (U) & $5(0-13)$ & $0(0-3)$ & $<0.001$ \\
24 hr FFP (U) & $0(0-9)$ & $0(0-2)$ & $<0.004$ \\
24 hr PLT (U) & $0(0-16)$ & $0(0-0)$ & $<0.007$ \\
\hline
\end{tabular}

Values are presented as number (\%) or median (interquartile range). ICU, intensive care unit; LOS, length of stay; pRBC, packed red blood cell; FFP, fresh frozen plasma; PLT, platelet. 
graphic and clinical characteristics were compared between groups. The suicide group was associated with higher fall heights and greater Injury Severity Scores (ISSs). Patients in this group were more likely to be younger and female relative to those in the accidental fall group. The mortality rate in the suicide group was higher relative to that in the accidental fall group (23.8\% vs. $6.5 \%$, $P=0.001$ ); however, relative to the suicide group, the accidental fall group showed a higher proportion of fatalities owing to brain damage (Table 2).

According to ISSs, the proportion of injuries to extremities with AIS scores of $\geq 3$ in the suicide group was higher relative to that in the accidental fall group ( $50.0 \%$ vs. $32.5 \%, P=0.031)$. According to AIS scores, the proportion of injuries to the lower extremities with an AIS score of $\geq 3$ in the suicide group was higher relative to that in the accidental fall group ( $52.4 \%$ vs. $31.5 \%$, $\mathrm{P}=$ 0.010); however, no injuries to the upper extremities with an AIS score of $\geq 3$ were recorded in the suicide group. The proportion of injuries to the abdomen with an AIS score of $\geq 3$ in the suicide group was higher relative to that in the accidental fall group (28.6\% vs. $13.0 \%, P=0.012$ ) (Fig. 1).
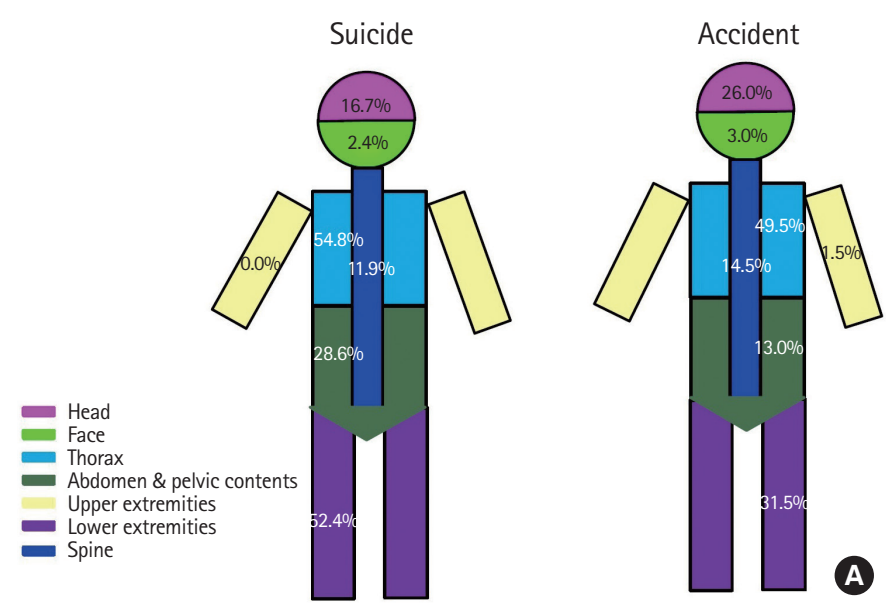

Table 3. Demographic, clinical, and fall-related characteristics of the survivor and non-survivor groups

\begin{tabular}{lccr}
\hline & $\begin{array}{c}\text { Survivor } \\
(n=219)\end{array}$ & $\begin{array}{c}\text { Non-survivor } \\
(n=23)\end{array}$ & P-value \\
\hline Age $(y r)$ & $45(34-53)$ & $40(34-56)$ & 0.934 \\
Sex, male & $178(81.3)$ & $14(60.9)$ & 0.030 \\
Fall height (m) & $6(6-10)$ & $10(7-15)$ & 0.002 \\
Intent (suicide) & $32(14.6)$ & $10(43.5)$ & 0.002 \\
Body mass index & $22.6(20.3-24.8)$ & $25.8(22.5-28.1)$ & 0.001 \\
Systolic blood pressure $(\mathrm{mmHg})$ & $120(105-140)$ & $98(82-122)$ & 0.002 \\
Heart rate (beat/min) & $86(78-98)$ & $111(95-125)$ & $<0.001$ \\
Shock index & $0.71(0.59-0.87)$ & $1.06(0.92-1.51)$ & $<0.001$ \\
Initial lactate (mmol/L) & $2.78(1.89-3.80)$ & $7.26(4.39-9.00)$ & $<0.001$ \\
Initial hemoglobin $(\mathrm{g} / \mathrm{dl})$ & $12.9(11.5-14.2)$ & $11.2(9.3-12.6)$ & 0.005 \\
Glasgow Coma Scale & $15(15-15)$ & $5(3-8)$ & $<0.001$ \\
Injury Severity Score & $17(13-25)$ & $34(29-41)$ & $<0.001$ \\
$\quad$ AIS head $\geq 3$ & $47(21.5)$ & $16(69.6)$ & $<0.001$ \\
AIS face $\geq 3$ & $5(2.3)$ & $2(8.7)$ & 0.135 \\
AIS thorax $\geq 3$ & $109(49.8)$ & $18(78.3)$ & 0.009 \\
AIS abdomen $\geq 3$ & $47(21.5)$ & $12(52.2)$ & 0.001 \\
AlS extremity $\geq 3$ & $80(36.5)$ & $6(26.1)$ & 0.320 \\
\hline
\end{tabular}

Values are presented as median (interquartile range) or number (\%). AIS, Abbreviated Injury Scale.

${ }^{a}$ Thirty six (35 in survivor/one in non-survivor) patients had no initial lactate level.

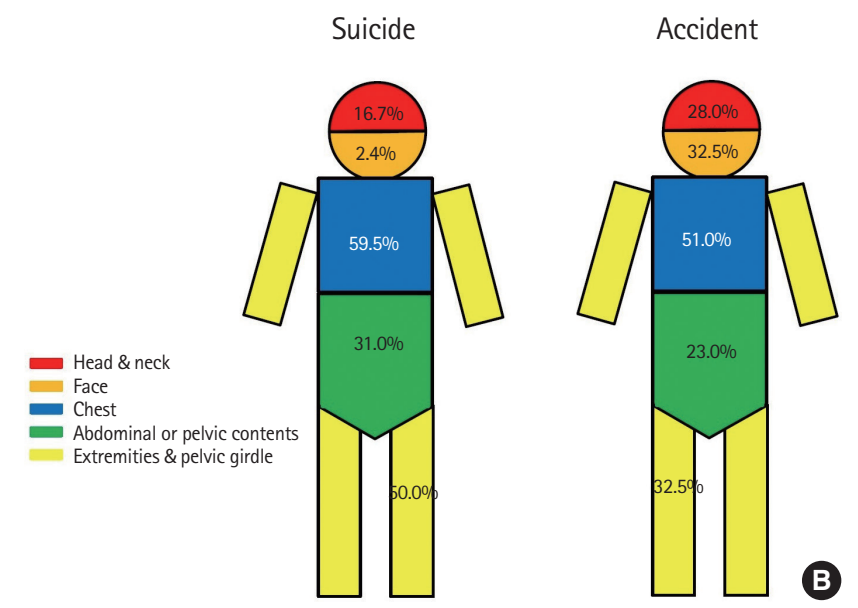

Fig. 1. Distribution of injury scores of $\geq 3$ in (A) the Abbreviated Injury Scale (divided by nine lesions) or (B) Injury Severity Score (divided by six lesions).

Table 4. Risk factors for mortality

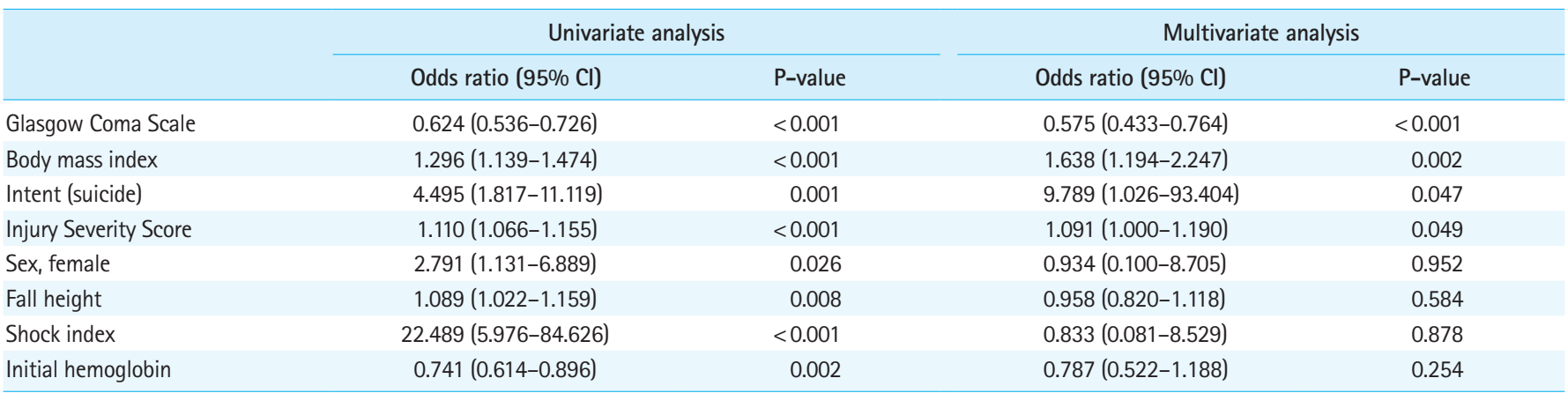

$\mathrm{Cl}$, confidence interval. 


\section{Survivors vs. non-survivors}

Fatalities were associated with higher ISSs. The non-survivor group included higher proportions of women and suicide intent and higher BMI values and fall heights relative to the survivor group (Table 3).

In the univariate analysis, Glasgow Coma Scale (GCS) scores, BMI values, suicide intent, ISSs, female sex, higher fall heights, shock index scores, and initial hemoglobin levels were statistically significant; therefore, they were included in the multivariate analysis. In this analysis, GCS score $(0.575 ; 95 \%$ confidence interval [Cl], 0.433-0.764; $\mathrm{P}<0.001)$, $\mathrm{BMI}$ value $(1.638 ; 95 \% \mathrm{Cl}, 1.194-$ 2.247; $P=0.002)$, suicide intent $(9.789 ; 95 \% \mathrm{Cl}, 1.026-93.404$; $\mathrm{P}=0.047)$, and ISS $(1.091 ; 95 \% \mathrm{Cl}, 1.000-1.190 ; \mathrm{P}=0.049)$ were identified as risk factors for mortality (Table 4).

\section{DISCUSSION}

In the current study, suicidal falls were associated with poorer outcomes and higher proportions of severe injuries to the abdomen and lower extremities relative to accidental falls. Suicide intent was a statistically significant risk factor for mortality in fall patients.

In a previous study, Choi et al. ${ }^{16}$ demonstrated that suicidal falls were associated with injuries of greater severity relative to those in accidental falls; however, fall heights in suicidal falls were higher relative to those in accidental falls. In contrast, Piazzalunga et al. ${ }^{9}$ reported that suicidal falls were associated with poorer outcomes relative to accidental falls, even with similar fall heights. Moreover, a national survey conducted in Pakistan revealed that casualties resulting from intentional falls were more likely to require hospital admission relative to those resulting from accidental falls; however, the study did not report fall heights. ${ }^{17}$ Similar to findings of previous studies, the suicide group in the current study showed higher mortality rates and higher ISS scores relative to those in the accident group. In previous studies, fall height was a critical factor affecting fall outcomes and was typically higher in suicidal relative to accidental falls, because individuals tended to select high locations from which to jump to their deaths, and falls from a low level are uncommon in suicidal patients. ${ }^{12}$ Consequently, the variability of fall height is an important factor, and the crude comparison made in the current study is insufficient from which to draw any firm conclusions. Although the minimum height of suicidal falls in this study was set at $5 \mathrm{~m}$, there is no established reference for this value. Detailed examination of the differences in outcomes according to fall height are warranted, as low-level falls are sometimes associated with mortality. ${ }^{15}$

Topp et al..$^{10}$ demonstrated that while suicidal fall patients sus- tained severe injuries, which determined their outcomes, there was no difference in prognoses between patient groups with comparable injury severity. Nevertheless, suicide intent and injury severity were both risk factors for mortality in the present study. Whether suicide intent determines injury severity remains unclear. However, as accidental falls tend to occur at workplaces or during leisure activities, they could have involved safety equipment, which would have mitigated the consequences of impact, ${ }^{16}$ resulting in varied injury severity with similar types of fall. In addition, suicide patients could have experienced psychosis or lacked sufficient treatment support from their families. ${ }^{18}$ Consistent with previous studies, BMI values and GCS scores were risk factors for mortality in the current study ${ }^{14}$; however, in contrast to other studies, fall height was not, ${ }_{1}^{12,16}$ which could be explained by the arbitrary minimum height imposed in the current study.

The injury pattern differed between the suicide and accident groups in the current study. In previous studies, suicidal falls were associated with injuries to the extremities.9.10,19 Teh et al. ${ }^{11}$ demonstrated that suicidal falls were associated with higher numbers of pelvic and lower limb fractures and fewer skull fractures relative to accidental falls. The current results are consistent with these results, in that the proportions of injuries to the lower extremities and abdomen in the suicide group were higher relative to those in the accidental fall group, while the proportion of head injuries in the accident group was higher relative to that in the suicide group. In addition, injuries to the upper extremities with AIS scores of $\geq 3$ were not recorded in the suicide group. In fact, patients in the suicide group tended to land feet first. Petaros et al. ${ }^{20}$ demonstrated that fall height was related to injury location. Skull fractures were associated with low-level falls, while thorax and extremity fractures were associated with high-level falls. Moreover, Casali et al. ${ }^{21}$ reported that, relative to falls from heights of $<12 \mathrm{~m}$, falls from heights of $\geq 12 \mathrm{~m}$ were associated with injuries of greater severity to the liver and diaphragm. These findings suggest that injury patterns could be associated with fall height; however, further research is required to elucidate the exact nature of this association.

The study was subject to several limitations. For example, patients who died before hospital admission were excluded from the analysis, which could have skewed the reported mortality rates. Autopsy is not routinely performed in South Korea; therefore, the details of injury locations or severity were unavailable for these individuals. In addition, suicide intent cannot be measured in patients who are deceased or have severe brain damage, therefore, they were excluded from the study. Further, as the minimum fall height was established arbitrarily, it could have affected the overall results. Although fall height was not a risk fac- 
tor for mortality in the current study, it is an important aspect of falls and is likely to affect outcomes. Moreover, this was a singlecenter study, and a nationwide dataset is required to provide more precise results.

In conclusion, suicidal falls were associated with poorer outcomes and the tendency to land feet first. GCS score, ISSs, BMI value, and suicide intent were identified as risk factors for mortality in high-level fall patients.

\section{CONFLICT OF INTEREST}

No potential conflict of interest relevant to this article was reported.

\section{REFERENCES}

1. Shin HY, Lee JY, Kim JE, et al. Cause-of-death statistics in 2016 in the Republic of Korea. J Korean Med Assoc 2018;61:573-84.

2. World Health Organization. The global burden of disease-estimates for 2000-2016. Geneva: World Health Organization; 2019.

3. Choi SB, Lee W, Yoon JH, Won JU, Kim DW. Risk factors of suicide attempt among people with suicidal ideation in South Korea: a cross-sectional study. BMC Public Health 2017;17: 579.

4. Jung K, Kim I, Park SK, et al. Preventable trauma death rate after establishing a national trauma system in Korea. J Korean Med Sci 2019;34:e65.

5. Kim H, Kwon SW, Ahn YM, Jeon HJ, Park S, Hong JP. Implementation and outcomes of suicide-prevention strategies by restricting access to lethal suicide methods in Korea. J Public Health Policy 2019;40:91-102.

6. Kim B, Ahn JH, Cha B, et al. Characteristics of methods of suicide attempts in Korea: Korea National Suicide Survey (KNSS). J Affect Disord 2015;188:218-25.

7. Byun CS, Park IH, Oh JH, Bae KS, Lee KH, Lee E. Epidemiology of trauma patients and analysis of 268 mortality cases: trends of a single center in Korea. Yonsei Med J 2015;56:220-6.

8. Aufmkolk M, Voggenreiter G, Majetschak M, Neudeck F, SchmitNeuerburg KP, Obertacke U. Injuries due to falls from a great height: a comparative analysis of injuries and their outcome following suicide-related and accidental falls. Unfallchirurg 1999;102:525-30.

9. Piazzalunga $D$, Ruberta $F$, Fugazzola $P$, et al. Suicidal fall from heights trauma: difficult management and poor results. Eur J Trauma Emerg Surg 2020;46:383-8.

10. Topp T, Muller T, Kiriazidis I, et al. Multiple blunt trauma after suicidal attempt: an analysis of 4,754 multiple severely injured patients. Eur J Trauma Emerg Surg 2012;38:19-24.

11. Teh J, Firth M, Sharma A, Wilson A, Reznek R, Chan O. Jumpers and fallers: a comparison of the distribution of skeletal injury. Clin Radiol 2003;58:482-6.

12. Alizo G, Sciarretta JD, Gibson S, et al. Fall from heights: does height really matter? Eur J Trauma Emerg Surg 2018;44:411-6.

13. James MK, Victor MC, Saghir SM, Gentile PA. Characterization of fall patients: does age matter? J Safety Res 2018;64:83-92.

14. Ren J, Waclawczyk A, Hartfield D, et al. Analysis of fall injuries by body mass index. South Med J 2014;107:294-300.

15. Turgut K, Sarihan ME, Colak C, Guven T, Gur A, Gurbuz S. Falls from height: a retrospective analysis. World J Emerg Med 2018; 9:46-50.

16. Choi JH, Kim SH, Kim SP, et al. Characteristics of intentional fall injuries in the ED. Am J Emerg Med 2014;32:529-34.

17. Fayyaz J, Wadhwaniya S, Shahzad H, et al. Pattern of fall injuries in Pakistan: the Pakistan National Emergency Department Surveillance (Pak-NEDS) study. BMC Emerg Med 2015;15 Suppl 2:S3.

18. Kelleher I, Ramsay H, DeVylder J. Psychotic experiences and suicide attempt risk in common mental disorders and borderline personality disorder. Acta Psychiatr Scand 2017;135:212-8.

19. Richter D, Hahn MP, Ostermann PA, Ekkernkamp A, Muhr G. Vertical deceleration injuries: a comparative study of the injury patterns of 101 patients after accidental and intentional high falls. Injury 1996;27:655-9.

20. Petaros A, Slaus M, Coklo M, Sosa I, Cengija M, Bosnar A. Retrospective analysis of free-fall fractures with regard to height and cause of fall. Forensic Sci Int 2013;226:290-5.

21. Casali MB, Battistini A, Blandino A, Cattaneo $C$. The injury pattern in fatal suicidal falls from a height: an examination of 307 cases. Forensic Sci Int 2014;244:57-62. 